\title{
Intermolecular double-quantum coherence MR microimaging of pig tail with unique image contrast
}

\author{
T. Hou ${ }^{\mathrm{a}}$, Z. Chen ${ }^{\mathrm{a}, \mathrm{b}}$, D.W. Hwang ${ }^{\mathrm{a}}$, J.H. Zhong ${ }^{\mathrm{c}}$, L.P. Hwang ${ }^{\mathrm{a}, *}$ \\ ${ }^{a}$ Department of Chemistry, National Taiwan University, and Institute of Atomic and Molecular Science, Academia Sinica, No. 1, Sec. 4, Roosevelt Road, \\ Taipei, Taiwan \\ ${ }^{\mathrm{b}}$ Departments of Physics and Chemistry, Xiamen University, and State Key Laboratory for Physical Chemistry of Solid Surface, Xiamen, Fujian 361005, \\ PR China \\ ${ }^{\mathrm{c}}$ Department of Radiology, University of Rochester Medical Center, Rochester, NY, USA
}

Received 25 October 2003; accepted 28 January 2004

\begin{abstract}
Image contrast in intermolecular double-quantum coherence (iDQC) imaging of a pig tail was investigated on a 7.05-T microimaging scanner. In addition to TR (repetition time) and TE (echo time), the time interval $\tau$ between radio frequency pulses during iDQC evolution and the areas under the iDQC-encode gradients in the iDQC imaging sequence were also used to manipulate image contrast. When suitable imaging parameters were selected, images with unique contrast, such as those with certain regions of the sample highlighted, were obtained without using contrast agents. The effects of iDQC-encode gradient on image contrast were studied quantitatively, and the unique contrast imposed by the related diffusion weighting was also shown. Experimental results demonstrated that the iDQC images have contrast fundamentally different from the conventional single-quantum coherence images. () 2004 Elsevier Inc. All rights reserved.
\end{abstract}

Keywords: MRI; Image contrast; Intermolecular double-quantum coherences; Microimaging; Diffusion-weighted

\section{Introduction}

In the past decade or so, great research efforts have focused on the phenomena of multiple spin echoes (MSEs) or intermolecular multiple-quantum coherences (iMQCs), which can be observed in a highly polarized spin system using a simple pulse sequence with two radio frequency (RF) pulses in the presence of pulsed field gradients [1,2]. MSEs and iMQCs have been analyzed theoretically using either classical dipolar field theory [1,3-7] or quantum mechanical density matrix treatments [1,8-11]. Although the formality of the two treatments is significantly different, so far both of them have led to the same quantitative predictions of the nuclear magnetic resonance (NMR) signals for simple pulse sequence, such as Cosy Revamped by Asymmetric Z-gradient Echo Detection (CRAZED) $[2,7,8]$. Recently, there has been great interest in application of the iMQC or MSE in magnetic resonance imaging (MRI) [12-

\footnotetext{
* Corresponding author. Tel.: +886-2-23668287; fax: +886-223620200.

E-mail address: nmr@gate.sinica.edu.tw (L.-P. Hwang).
}

16], due to the unique contrast mechanisms of iMQCs that might potentially result in novel image contrast, and provide improved detection of tumors, for example, without the need for contrast agent injection. Warren and coworkers $[9,10,12,13]$ first proposed an intermolecular zero-quantum coherence (iZQC) imaging method. The contrast of iZQC images reveals structural features, which are not seen in conventional MR images. The first in vivo iDQC image was explored by Mori et al. [15]. Zhong and coworkers successfully obtained the first multislice human brain iDQC images using a 1.5-T clinical scanner [17], and demonstrated the feasibility of iDQC imaging at $1.5 \mathrm{~T}$ for human brain functional mapping [18]. They have also investigated theoretically and experimentally, diffusion and relaxation behaviors of iMQCs related to MR imaging contrast [19-22]. Novel MR imaging based on a combined diffusion weighting and iDQC encoding [23], and dependence of image contrast in iDQC images of human brain on several time variables have been reported [24].

Warren and coworkers [2] have shown that iZQC signals originate primarily from coupled spins separated by a macroscopic length, the so-called correlation distance $d_{c}=$ 

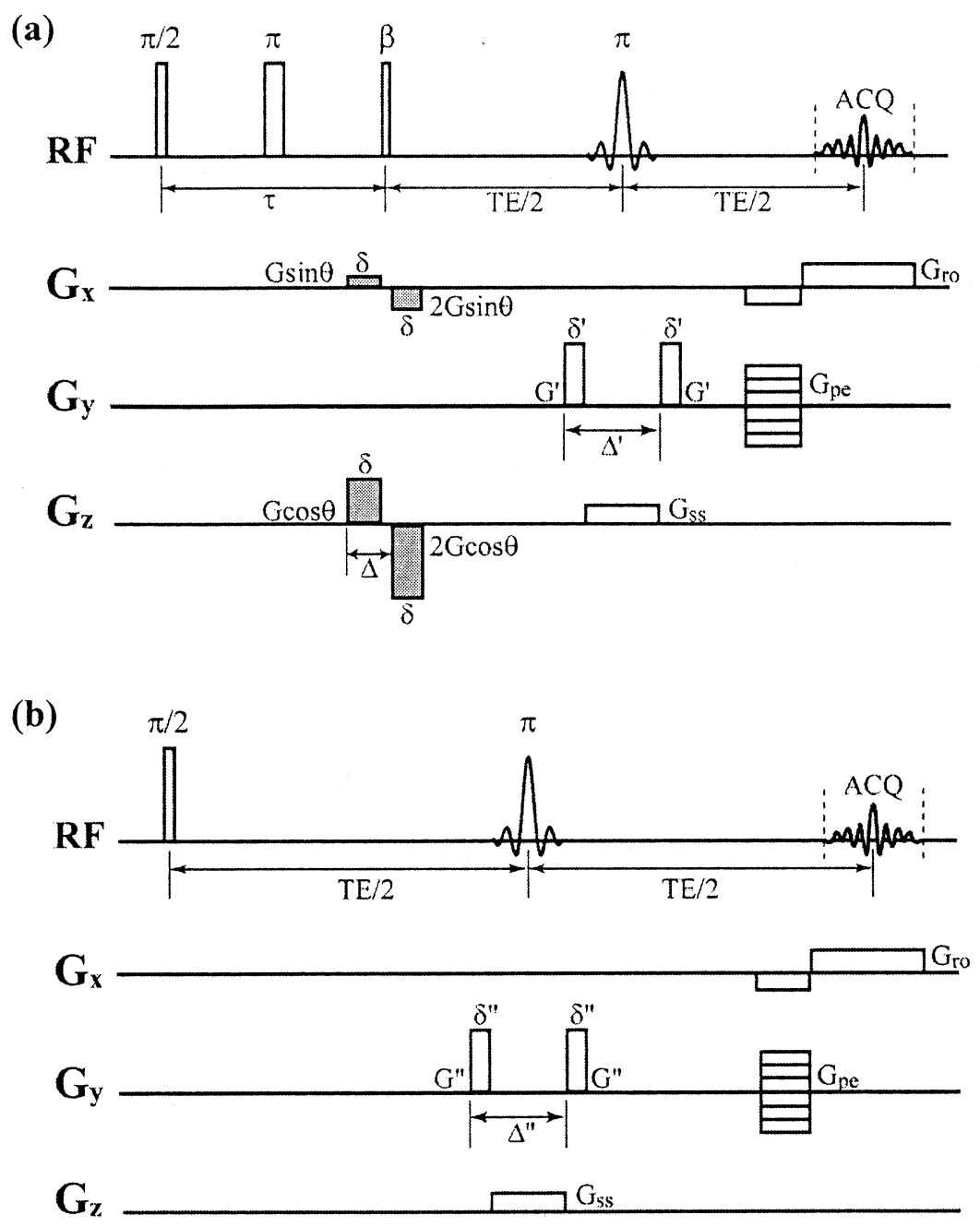

Fig. 1. (a) iDQC SE imaging sequence, (b) diffusion-weighted SE imaging sequence.

$\pi /(\gamma G \delta)$, where $\gamma$ is the gyromagnetic ratio, $G$ and $\delta$ are the strength and duration of the iZQC-encode gradient in CRAZED sequence, or half a cycle of the magnetization helix generated by the coherence-selection gradient. This correlation distance $d_{c}$ is produced by the first iMQC-encode gradient in a CRAZED-like sequence, so the signal from iDQCs generated with such pulse sequence should have similar spatial dependence as that from iZQCs. Several research groups [25-28] have shown theoretically and experimentally that the iDQC images of heterogeneous phantoms do reveal altered contrast. For instance, Capuani and coworkers $[29,30]$ have demonstrated recently that by varying the correlation distance, in vivo and ex vivo iMQC images reveal the characteristics of trabecular bone structures of particular sizes. Therefore, tuning the correlation distance may provide new contrast for iMQC imaging. However, to the best of our knowledge, to date there are few practical applications of such unique contrast for iMQC imaging.

In the present work, iDQC images of an isolated pig tail were obtained at $7.05 \mathrm{~T}$, and the effects of longitudinal relaxation, transverse relaxation, diffusion and correlation distance on the iDQC image contrast were investigated. With careful selection of imaging parameters, unique contrast was obtained without the need to use contrast enhance agents, and parts of the pig tail with different morphological features were selectively highlighted.

\section{Methods and materials}

The spin echo (SE) CRAZED-like imaging sequence, as shown in Fig. 1a, was used to obtain iDQC images. The first $\pi / 2$ pulse creates iMQCs, including iZQC, iDQC and other high-order quantum coherences, and then the iMQC-encode gradient pair with a total area ratio of $1: 2$ before and after the $\beta$ RF pulse was applied to select the desired iDQCs. The $\beta$ pulse then transforms the iDQC signals into detectable signal quantum coherence (SQC) signals. In iDQC imaging, $\beta$ is chosen to be either a $\pi / 3$ (for $\mathrm{n}=-2$ quantum transition) or a $\pi / 3$ (for $\mathrm{n}=+2$ quantum transition) pulse for maximum iDQC signal intensities, which is increased by 
a factor of $3 \sqrt{ } 3 / 4$ compared to when $\beta$ is set to be $\pi / 2$ [17]. In the present work, $\pi / 3$ pulse with $n=-2$ was used for the $\beta$ RF pulse to take advantage of the more uniform excitation with a smaller flip angle. In the iDQC sequence as shown in Fig. 1a, the first $\pi$ pulse refocuses chemical shifts and magnetic field inhomogeneities while retaining long-range dipolar couplings. Besides removing magnetic field inhomogeneity and chemical shift effects, the second $\pi$ pulse (a soft Sinc pulse) also acts as the slice selection pulse. Crusher gradients $G^{\prime} \delta^{\prime}$ were placed along the $y$-axis on either side of the soft $\pi$ pulse to dephase signals other than that from the selected slice. The phase encoding gradient and dephasing pulses for the readout gradient were placed right before acquisition to minimize their interactions with coherence selections. Due to the background gradients and unavoidable imperfections in RF pulse flip angles and gradient waveforms, phase cycling of RF pulses are often necessary. A four-step phase cycling scheme $(x,-x, y,-y)$ for the first $\pi / 2$ RF pulse along with $(x, x,-x,-x)$ for the receiver phase, were adopted to remove residual conventional SQC signals [31,32]. Charles-Edwards et al. have demonstrated that the use of appropriate phase cycling smoothes signal dips in MSE experiments, which was caused by residual conventional SQCs [33]. iDQC images with and without the phase cycling schemes were acquired in this study, and the image quality improvement imposed by the phase cycling will be illustrated in the next section.

In conventional SE imaging sequence, TR (repetition time) and TE (echo time) are the two time parameters that are usually used to obtain images with different contrast effects based on $\mathrm{T}_{1}$ or $\mathrm{T}_{2}$-weighting. Besides $\mathrm{TR}$ and $\mathrm{TE}$, more experimental parameters, such as the areas of the iDQC-encode gradient and time interval $\tau$ between the first $\pi / 2$ and $\beta$ RF pulses can be adjusted in the iDQC imaging sequence as shown in Fig. 1a. Therefore, there is more flexibility in the $\mathrm{iDQC}$ sequence, and unique contrast in the iDQC images may be achieved more readily. Although both $\mathrm{T}_{1}$-weighted $\mathrm{iDQC}$ and conventional SE images are obtained by varying TR, longitudinal relaxation related to iDQC signals is not a monoexponential process [21], which is different from the conventional SQC. It was observed that $\mathrm{T}_{1}$-weighted iDQC images show more pronounced contrast than that of $\mathrm{T}_{1}$-weighted $\mathrm{SQC}$ images (details will be given in the next section). In both iDQC and conventional SE imaging sequence, TE can be varied to achieve $\mathrm{T}_{2}$-weighted images. However, the iDQC signal right after the $\beta \mathrm{RF}$ pulse needs certain time to grow to its maximum, the peak strength and the rate to reach the maximum are functions of $\mathrm{T}_{2}$ values [22], so the variation of iDQC signal with respect to the increase of TE will also be different from that of SQC signals. Therefore, the combination of the iDQC signal growth and the transverse relaxation process related to intermolecular dipolar interactions will certainly aid in the creation of unique imaging contrast. Moreover, $\tau$ in the iDQC imaging sequence is a unique time variable, which is not present in the SE sequence. This additional time variable will add extra means to manipulate iDQC image contrast.

In addition, iDQC-encode gradient pair ( $G \delta$ and $2 G \delta$ ) and the crusher gradient pair $\left(G^{\prime} \delta^{\prime}\right)$ are another two parameters that could be used as additional tuning tools for iDQC imaging contrast variant. Besides their functions of encoding iMQCs and dephasing unwanted signals outside the selected slice, respectively, the presence of these gradients also encodes molecular diffusion process. By varying the areas of these gradients, images of different diffusionweighting are obtained, and the relationship between the signal intensity and the gradient area can be quantitatively characterized. However, since the iDQC-encode gradient acts on iDQCs, while the crusher gradient acts on SQCs converted from iDQCs by the $\beta$ RF pulse, the efficiency of these gradients for diffusion weighting are different. As demonstrated previously by Zhong and coworkers [20,23], the diffusion rate of iDQCs in the evolution period is twice of that of conventional SQCs. Therefore, the diffusionweighting effect imposed by iDQC-encode gradient is twice of the crusher gradient. In addition to its effects on diffusion, variation of the iDQC-encode gradient area can also impose changes in correlation distance $d_{c}$, which may also contribute to the image contrast variation, as suggested previously [25-28]. For comparison, imaging pulse sequence as shown in Fig. $1 \mathrm{~b}$ was used to obtain conventional SQC diffusion-weighted SE images, and the corresponding parameters were set to the same as those in diffusionweighted iDQC imaging experiments. The contrast variations were characterized quantitatively for diffusionweighted SE images, iDQC-encode gradient imposed and crusher gradient imposed diffusion-weighted iDQC images.

All experiments reported here were performed using a 7.05-T Varian Unity Inova NMR spectrometer (Varian, Palo Alto, CA, USA) with microimaging capabilities. The images were obtained at room temperature with a microimaging probehead (Resonance Research Inc., Millerica, MA, USA), which consists of a quadrature birdcage imaging RF coil (30 mm i.d.) and a self-shielded gradient system with peak gradient strength of $100 \mathrm{G} / \mathrm{cm}$ each in $x$-, $y$ - and $z$-directions. Pig tail samples were purchased from the local meat market, cut into sections of about $4 \mathrm{~cm}$ long and kept refrigerated at $\sim 5^{\circ} \mathrm{C}$ until measured. The following imaging parameters were used for both iDQC and conventional SE images: field-of-view of $3.5 \mathrm{~cm} \times 3.5 \mathrm{~cm}$, slice thickness of $3 \mathrm{~mm}$, matrix size of $256 \times 128$. In the $\mathrm{iDQC}$ imaging experiments, the durations of the hard $\pi / 2$ pulse, the $\beta$ pulse, the hard $\pi$ pulse and the soft Sinc pulse were $29.5 \mu$ s, $17.9 \mu \mathrm{s}, 59 \mu \mathrm{s}$ and $2 \mathrm{~ms}$, respectively. In experiments other than the diffusion-weighted imaging, the iDQC-encode gradient strength $\mathrm{G}$ of $21.4 \mathrm{G} / \mathrm{cm}$, duration $\delta^{\prime}$ of $1 \mathrm{~ms}$, and the crusher gradient strength $G^{\prime}$ of $24.4 \mathrm{G} / \mathrm{cm}$, duration $\delta^{\prime}$ of 0.5 $\mathrm{ms}$ were used, while in diffusion-weighted imaging experiments, the iDQC-encode gradient strength $G$ and the crusher gradient strength $G^{\prime}$ were both set to $42.8 \mathrm{G} / \mathrm{cm}$, and the gradient durations were varied from $0.5 \mathrm{~ms}$ to $2.5 \mathrm{~ms}$ in 

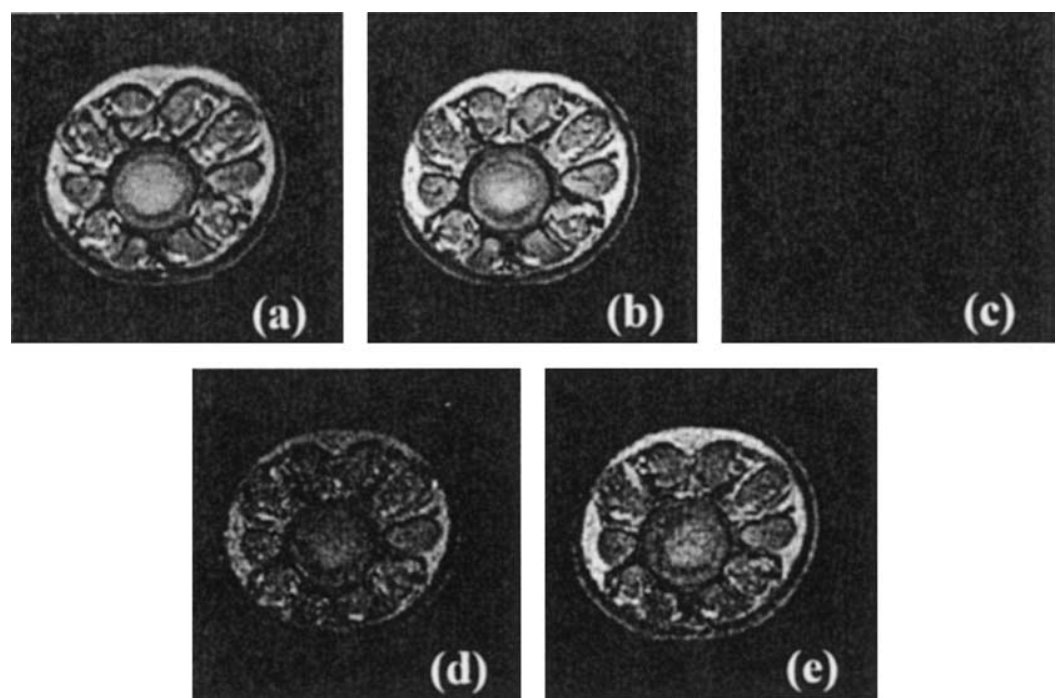

Fig. 2. Images of a pig tail under different experimental schemes. Conventional SE image (a); iDQC images with four-step phase cycling when the iDQC-encode gradients are along the direction of the static magnetic field $\mathrm{B}_{0}$ (b), along the direction of the magic angle (c) and along the direction perpendicular to $\mathrm{B}_{0}(\mathrm{~d})$; iDQC image with the same imaging parameters as in (b) but without phase cycling.

step of $0.5 \mathrm{~ms}$. Because a very high-level suppression of the conventional SQC signal was realized using the iDQC imaging sequence, the individual receiver gain was adjusted to fully utilize the dynamic range of the analog-to-digital converters. In order to quantitatively evaluate the signal intensities of different regions of the pig tail sample, receiver gain was set to the same value in the experiments of varying the iDQC-encode and crusher gradient areas. The same treatment was used for the diffusion-weighted SE imaging.

\section{Results and discussions}

At the center of the pig tail is a central spinal bone full of bone marrow, which is surrounded by tendons, muscles, fat and subcutaneous tissue, and the outmost layer is skin. A transverse image of the pig tail obtained by conventional SE MRI with TR of $4 \mathrm{~s}$ and TE of $35 \mathrm{~ms}$ is shown in Fig. 2a. The marrow in the central spinal bone, the muscles and the fat and subcutaneous tissues all appear bright. The skin surrounding the tail has low intensity, and the outer layer of the bone is dark. iDQC images were also obtained using the sequence shown in Fig. 1a. To verify that the signals in iDQC images are indeed from $\mathrm{DDQC}$ and not from a leakage of SQCs through the iDQC-encode gradients, experiments were performed with the iDQC-encode gradients applied along the static magnetic field $\left(\mathrm{B}_{0}\right)$ direction, along the magic angle $\left(54.7^{\circ}\right)$ with respect to $B_{0}$, and along the direction perpendicular to $\mathrm{B}_{0}$ ( $x$-direction in this case). Time durations with TR of $4 \mathrm{~s}, \tau$ of $2.7 \mathrm{~ms}$ and TE of $35 \mathrm{~ms}$ were used in acquiring these images. The resulting images are shown in Fig. $2 \mathrm{~b}-$ d. As expected, a signal minimum was observed when the gradients are at the magic angle (Fig. $1 \mathrm{c})$, and the signal is approximately half when the gradients are along the direction perpendicular to $B_{0}$ (Fig. 1d), in comparison to the image with iDQC gradients along $\mathrm{B}_{0}$ (Fig. 1b). However, when the iDQC-encode gradients are at the magic angle, there are still weak residual signals, not zero as predicted theoretically. This is because the pig tail is structurally inhomogeneous and there exists magnetic susceptibility variation across the sample. As a result, the actual gradient at the sample, which is a combination of the applied iDQC-encode gradient and local gradient, deviates slightly from the magic angle across the sample.

To demonstrate the image quality improvement imposed by the four-step phase cycling scheme, $(x,-x, y,-y)$ for the first $\pi / 2 \mathrm{RF}$ pulse and $(x, x,-x,-x)$ for the receiver, iDQC images obtained with and without the phase cycling scheme are shown in Figs. $2 b$ and $2 e$, respectively. For the image without the phase cycling scheme, the phase scheme $(x, x, x$, $x$ ) was applied for both the first $\pi / 2 \mathrm{RF}$ pulse and the receiver. Except the phase cycling scheme difference, all the other imaging parameters were exactly the same for the two images. As the images show clearly, the phase cycling scheme does reduce noise level and improves signal-tonoise ratio of the images. Experimental results show that the iDQC image with phase cycling appears smoother and cleaner.

To investigate the unique contrast created by iDQC imaging, images of the pig tail sample obtained using iDQC imaging sequence with different time variables, TR, $\tau$ and TE, along with SE images with corresponding TR and TE values are shown in Fig. 3. Fig. 3a is an iDQC image with TR of $4 \mathrm{~s}, \tau$ of $2.7 \mathrm{~ms}$, and TE of $35 \mathrm{~ms}$ (the same image as in Fig. 2b). Longitudinal relaxation times of the pig tail sample are in the range of $0.5 \sim 1.3 \mathrm{~s}$. Therefore, TR of $4 \mathrm{~s}$ is sufficiently long to avoid $\mathrm{T}_{1}$-weighting effects. TE of 35 ms was chosen such that iDQC signals from different tis- 

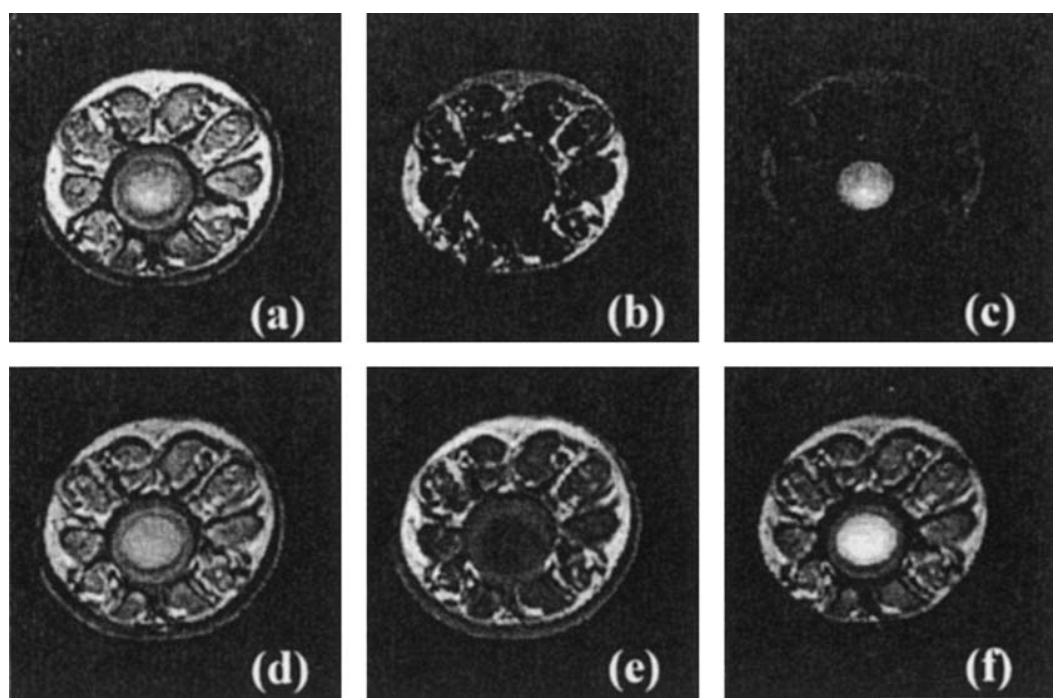

Fig. 3. iDQC images of a pig tail with different time parameters. (a) $\mathrm{TR}=4 \mathrm{~s}, \tau=2.7 \mathrm{~ms}$ and $\mathrm{TE}=35 \mathrm{~ms}$; (b) $\mathrm{TR}=0.3 \mathrm{~s}, \tau=2.7 \mathrm{~ms}$ and $\mathrm{TE}=14$ $\mathrm{ms}$ and (c) TR $=2.5 \mathrm{~s}, \tau=50 \mathrm{~ms}$ and $\mathrm{TE}=70 \mathrm{~ms}$ in which bone marrow in the central spinal bone is highlighted. For comparison, (d) $-(\mathrm{f})$ are the corresponding conventional SE images with the same TR and TE values as in (a)-(c), respectively.

sues are near their maxima. Fig. 3a shows that iDQC image obtained using these settings does not provide much additional feature, with image contrast similar to that of the SE image (Fig. 3d) of the same sample using the corresponding settings of $\mathrm{TE}=35 \mathrm{~ms}$ and $\mathrm{TR}=4 \mathrm{~s}$.

Figures $3 \mathrm{~b}$ and $3 \mathrm{e}$ are $\mathrm{T}_{1}$-weighted $\mathrm{iDQC}$ and $\mathrm{SE}$ images (TR $=0.3 \mathrm{~s})$, respectively, of the same sample. Signal from fat tissues has a higher intensity, because $T_{1}$ of fat is usually shorter than other tissues such as muscles. Although the same TR was used in both Figs. $3 \mathrm{~b}$ and $3 \mathrm{e}$, the contrast in the two images is not the same. In the $\mathrm{T}_{1}$-weighted iDQC image, only fat tissue is clearly seen with a strong positive contrast to the surrounding tissues. In the $\mathrm{T}_{1}$-weighted SE image, however, although fat tissue has a higher intensity, muscles, skin and even part of bone marrow are still visible. The contrast difference between the two images is primarily due to the different longitudinal relaxation characterization related to intermolecular dipolar interactions and to conventional SQCs. Unlike the monoexponential longitudinal relaxation process of SQCs, with the signal following the function of the form $\left(1-e^{-T R / T_{1}}\right)$, the longitudinal relaxation process related to iDQC dipolar interactions is $(1-$ $\left.e^{-T R / T_{1}}\right)^{2}[21]$. This indicates that when intermolecular dipolar interactions are involved, the recovery of the longitudinal magnetization through longitudinal relaxation process is slower than when only conventional single-quantum coherences are involved. As a result, the $\mathrm{T}_{1}$ difference in different tissues of the pig tail sample are amplified in iDQC images, and their contrast is greater than that in conventional SQC images.

Figure $3 \mathrm{c}$ is an iDQC image with the central spinal bone marrow being highlighted. This iDQC image was obtained using the time variables: $\mathrm{TR}=2.5 \mathrm{~s}, \tau=50 \mathrm{~ms}$ and $\mathrm{TE}=$ $70 \mathrm{~ms}$. For comparison, conventional SE image with $\mathrm{TR}=$ $2.5 \mathrm{~s}$ and $\mathrm{TE}=70 \mathrm{~ms}$ is shown in Fig. 3f, in which the central spinal bone marrow also has a higher intensity, but the surrounding fat tissues and muscles are still visible and with considerable intensities. The contrast enhancement of iDQC images benefits from the presence of the additional time variable $\tau$.

Figures $4 \mathrm{a}$ and $4 \mathrm{~b}$ are iDQC images of the pig tail with varied areas of the iDQC-encode gradient or the crusher gradient, while keeping their gradient strengths, $G$ and $G^{\prime}$ constant at $42.8 \mathrm{G} / \mathrm{cm}$. In Fig. 4a, the duration $\delta^{\prime}$ of the crusher gradient was kept constant at $0.5 \mathrm{~ms}$, and the duration $\delta$ of iDQC-encode gradient was set to $0.5 \mathrm{~ms}, 1.0 \mathrm{~ms}$, $1.5 \mathrm{~ms}, 2.0 \mathrm{~ms}$ and $2.5 \mathrm{~ms}$, respectively. In Fig. $4 \mathrm{~b}$, the duration $\delta$ of the iDQC-encode gradient was kept constant at $0.5 \mathrm{~ms}$, but the duration $\delta^{\prime}$ of the crusher gradient was set to $0.5 \mathrm{~ms}, 1.0 \mathrm{~ms}, 1.5 \mathrm{~ms}, 2.0 \mathrm{~ms}$ and $2.5 \mathrm{~ms}$, respectively. Figure $4 c$ is SE images of the same sample using the sequence in Fig. 1b with the gradient strength $G^{\prime \prime}$ kept constant at $42.8 \mathrm{G} / \mathrm{cm}$, while the gradient duration $\delta^{\prime \prime}$ set to $0.5 \mathrm{~ms}, 1.0 \mathrm{~ms}, 1.5 \mathrm{~ms}, 2.0 \mathrm{~ms}$ and $2.5 \mathrm{~ms}$, respectively. In Fig. $4, \mathrm{TR}=3 \mathrm{~s}$ and $\mathrm{TE}=35 \mathrm{~ms}$ were used for both the iDQC and conventional SE images, and $\tau=6 \mathrm{~ms}$ was used for all iDQC images. These images clearly show that the signal intensities decrease with the increase of corresponding gradient area. This is expected with the gradient-imposed diffusion effects, larger gradient areas making the diffusion effects greater, thus resulting in lower signal intensities. It is noticed that the bone marrow has faster signal decay than that of the fat tissue in all three sets of images, therefore, a contrast variation is present with the increase of gradient areas. This is because fat tissues have a smaller diffusion coefficient. Besides these differences, it is also obvious that iDQC images with variation of the iDQCencode gradient area demonstrate a greater contrast change than those of both the iDQC images with variation of the 
(a)

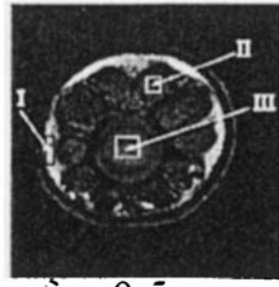

$\delta=0.5 \mathrm{~ms}$

(b)

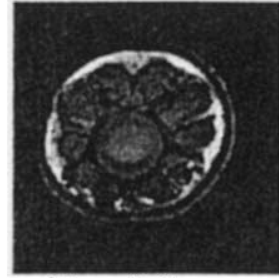

$\delta^{\prime}=0.5 \mathrm{~ms}$

(c)

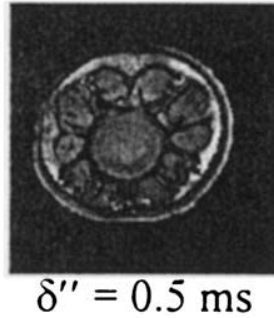

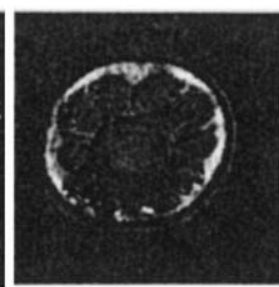

$1.0 \mathrm{~ms}$

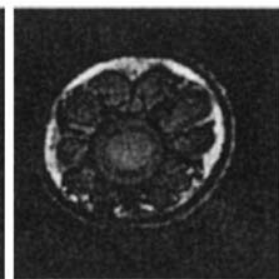

$1.0 \mathrm{~ms}$

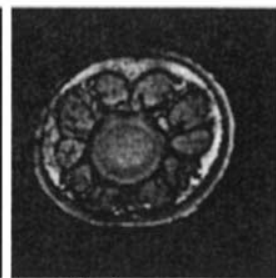

$1.0 \mathrm{~ms}$

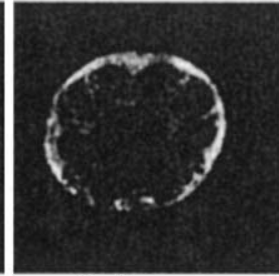

$1.5 \mathrm{~ms}$

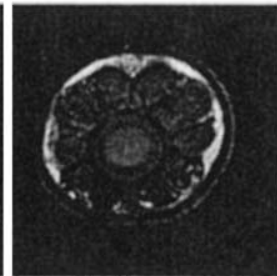

$1.5 \mathrm{~ms}$

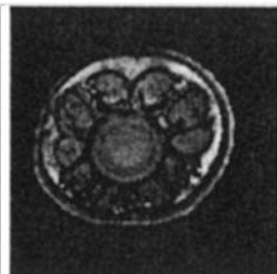

$1.5 \mathrm{~ms}$

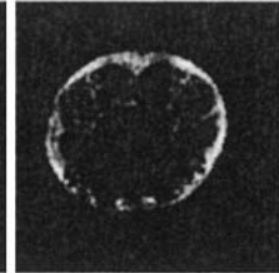

$2.0 \mathrm{~ms}$

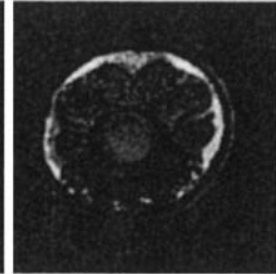

$2.0 \mathrm{~ms}$

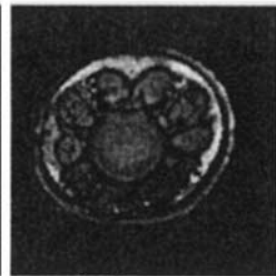

$2.0 \mathrm{~ms}$

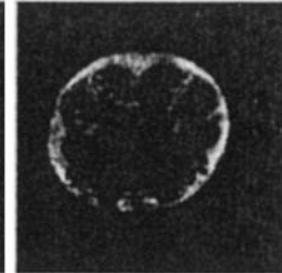

$2.5 \mathrm{~ms}$

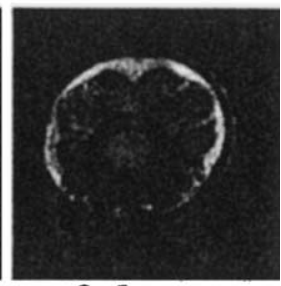

$2.5 \mathrm{~ms}$

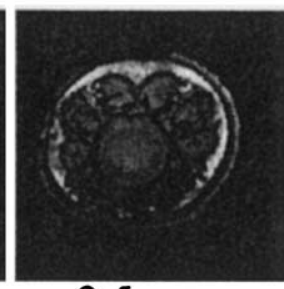

$2.5 \mathrm{~ms}$

Fig. 4. Diffusion-weighting images of a pig tail. (a) iDQC image with different iDQC-encode gradient duration $\delta$, (b) iDQC images with different crusher gradient a duration $\delta^{\prime}$ and (c) conventional diffusion-weighted SE images with different gradient duration $\delta^{\prime \prime}$.

crusher gradient area and conventional diffusion-weighted SE.

To characterize the contrast change quantitatively, three different regions of interest depicted by the three squares labeled I, II and III in the first image of Fig. 4a were used to measure mean signal intensities of fat tissue, muscle and bone marrow, respectively. The normalized signal intensities were then plotted as a function of the diffusion weighting factor $b$, which is defined by $b=\gamma^{2} G^{2} \delta^{2}(\Delta-\delta / 3)$, where $\Delta$ is the diffusion time. For diffusion-weighted SE images, $b=\gamma^{2} G^{\prime \prime 2} \delta^{\prime \prime 2}\left(\Delta^{\prime \prime}-\delta^{\prime \prime} / 3\right)$ and for iDQC images with varied crusher gradient duration, $b=\gamma^{2} G^{\prime 2} \delta^{\prime 2}\left(\Delta^{\prime}-\right.$ $\left.\delta^{\prime} / 3\right)$. It is noted that for iDQC images with varied iDQCencode gradient duration, the gradient pair has a 1:2 ratio in strength. As reported in our previous article [20], the apparent diffusion coefficient during the evolution period satisfies $D_{2}^{a p p}=2 D_{T}$, in which $D_{T}$ is the conventional translational diffusion coefficient. Therefore, the action of the first gradient, $G \delta$ results in the diffusion weighting factor of $b=2 \gamma^{2} G^{2} \delta^{2}(\Delta-\delta / 3)$. On the other hand, the action of the second gradient, $2 G \delta$, results in the diffusion weighting factor of $b=\gamma^{2}(2 G \delta)^{2}(\Delta-\delta / 3)=4 \gamma^{2} G^{2} \delta^{2}(\Delta-\delta / 3)$ since the iDQC terms are already converted to SQC signals by the $\beta$ RF pulse during the detection period. To take two sides into consideration, an averaged diffusion weighting factor, $b$ $=3 \gamma^{2} G^{2} \delta^{2}(\Delta-\delta / 3)$, was approximately used for iDQC images with the varied iDQC-encode gradient areas. The plot of the normalized signal intensities versus the diffusion weighting factors is shown in Fig. 5. The plot shows that in the diffusion-weighted SE images, as the diffusion weighting factor $b$ increases from 8 to $330 \mathrm{~s} / \mathrm{mm}^{2}$, the signal intensity of the fat tissue decreases to about $66 \%$, the muscles to about $67 \%$, and the bone marrow to about $63 \%$, with three different tissues therefore having about the same level of signal decay. In the iDQC images with the crusher gradient duration variation, the signal of fat decreases to about $58 \%$, bone marrow to about $57 \%$ and muscles to about $65 \%$, as $b$ increases from 8 to $330 \mathrm{~s} / \mathrm{mm}^{2}$. Although fat and bone marrow have a slightly faster signal decaying than muscle, the difference is not significant. The diffusion of iDQC images due to crusher gradient appears slight faster than in diffusion-weighted SE images. In the iDQC images with iDQC-encode gradient duration variation, the signal decreasing is still present, but the decay rates are quite different for different tissues: fat tissue reduces to about $63 \%$, muscle to about $28 \%$ and bone marrow to about $17 \%$, as the diffusion weighting factor $b$ increases from 8 to $330 \mathrm{~s} / \mathrm{mm}^{2}$. These results clearly suggest that the contrast in the iDQC images with iDQC-encode gradient area variation is more significant than both iDQC images with the crusher gradient area variation and the conventional diffusion-weighted SE images. This can be partially explained by the fact that the 


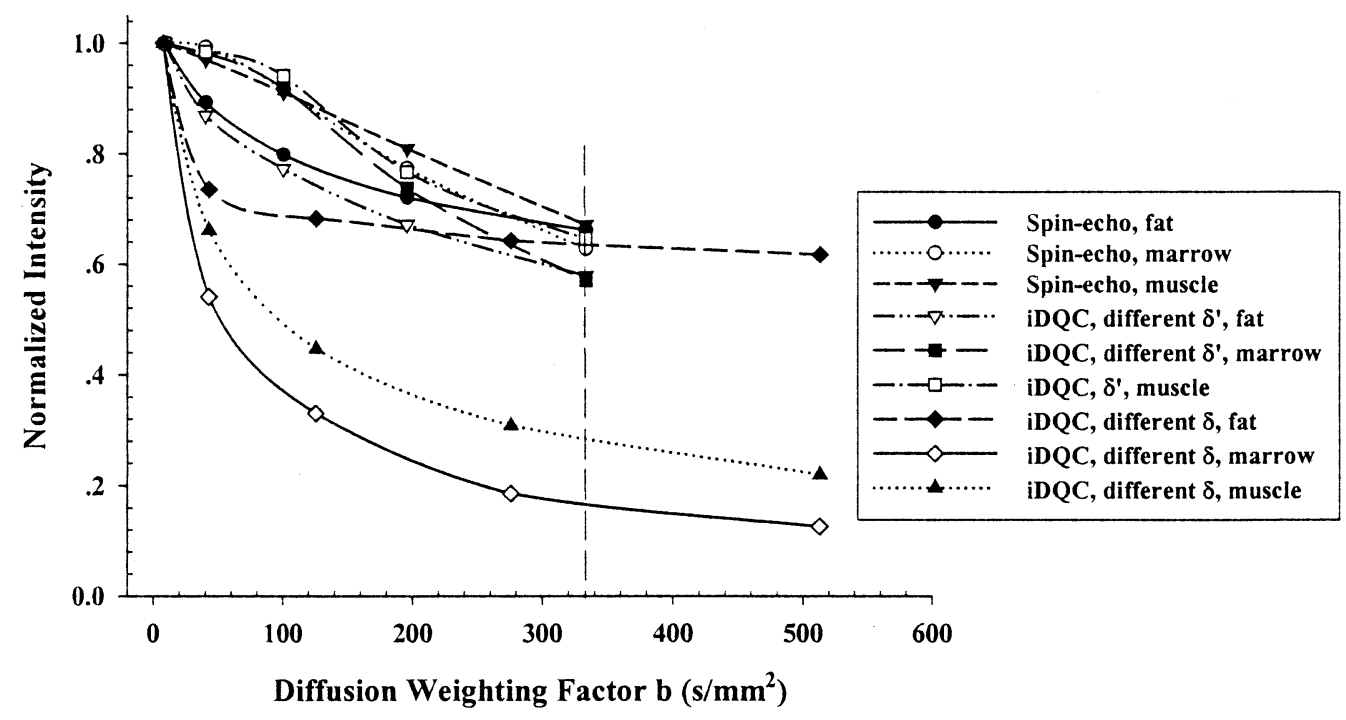

Fig. 5. Plot of normalized signal intensities of conventional SE images, iDQC images with crusher gradient duration variation and iDQC-encode gradient duration variation as a function of the diffusion weighting factor $b$. In each image, signal intensities of three regions of interest corresponding to fat, muscle and bone marrow depicted by I, II and III in the first image of Fig. 4a were obtained.

apparent diffusion coefficient of iDQCs signals is twice as fast as that of conventional SQC signals, therefore, diffusion weighting is more significant when iDQC signals are involved. However, the variation of the iDQC-encode gradient areas also induces changes in correlation distance, $d_{c}$. The greater the gradient area, the smaller the correlation distance. As the studies [25-27,29,30] have shown that iMQC image contrast does depend on the correlation distances, contribution of the correlation distance in the contrast of iDQC image due to variation of the iDQC-encode gradient area cannot be ignored. Correlation distances usually induce novel contrast in heterogeneous samples $[13,29]$. Therefore, image contrast of biological samples with more structural features should be more sensitive to correlation distance variation. In the pig tail used in the present study, fat, muscle and bone have different structural features. The spinal tail bone consists of complex threedimensional bone trabeculae [29], muscle has fibers, which may be regarded as a two-dimensional structural feature, and fat is more homogenous and has the least structural features among the three. These differences in microstructures may explain why upon the variation of iDQC-encode gradient the images of bone marrow and muscle show much greater change than that of fat, and bone marrow is the one with the greatest change among the three components. To evaluate quantitatively the contribution of the correlation distance to the contrast enhancement, we are currently carrying out more detailed studies, and results will be presented in a separate report.

\section{Conclusion}

In this article, iDQC image contrast of a pig tail sample based on CRAZED-like SE imaging sequence was investi- gated using a 7.05-T microimaging scanner. Unlike conventional MRI, in which image contrast is based on variations in spin density and/or relaxation times, iDQC has more variables to be manipulated to obtain unique image contrast. In addition to TR and TE, experimental parameters, such as time interval of $\tau$ and the areas of the iDQC-encode gradient or the crusher gradient in the iDQC imaging sequence, can be used as tuning tools for image contrast. When suitable imaging parameters are selected, images with unique contrast, such as those with certain regions of the sample highlighted, can be achieved. The effects of iDQC-encode gradient on image contrast were also studied quantitatively. Diffusion weighting imposed by the iDQC-encode gradient certainly contribute to the contrast, however, the contribution of correlation distance cannot be ruled out. Questions regarding the role of the correlation distance and how much it contributes to contrast remain to be answered through more detailed studies, which is underway in our laboratories.

\section{Acknowledgments}

This project is partially supported by the program for Promoting Academic Excellence of Universities of Taiwan, NNSF of China under Grant 10234070, and the NIH under Grant NS41048.

\section{References}

[1] Deville G, Bernier M, Delrieux JM. NMR multiple echoes observed in solid ${ }^{3} \mathrm{He}$. Phys Rev B 1979;19:5666-87.

[2] Warren WS, Richter W, Andreotti AH, Farmer BT. Generation of impossible cross-peaks between bulk water and biomolecules in solution NMR. Science 1993;262:2005-9. 
[3] Bowtell R, Bowley RM, Glover P. Multiple spin echoes in liquids in a high magnetic field. J Magn Reson 1990;88:643-51.

[4] Jeener J, Vassenbroek A, Broekaert P. Unified derivation of the dipolar field and relaxation terms in the Bloch-Redfield equations of liquid NMR. J Chem Phys 1995;103:1309-32.

[5] Vlassenbroek A, Jeener J, Broekaert P. Macroscopic and microscopic fields in high-resolution liquid NMR. J Magn Reson A 1996;118: 234-46.

[6] Broekaert P, Vlassenbroek A, Jeener J, Lippens G, Wieruszeski JM. Observation and selective suppression of the dipolar-field effects in 2D NMR in liquid in homogeneous fields. J Magn Reson A 1996; 120:97-104.

[7] Levitt MH. Demagnetization field effects in two-dimensional solution NMR. Concepts Magn Reson 1996;8:77-103.

[8] Lee S, Richter W, Vathyam S, Warren WS. Quantum treatment of the effects of dipolar-dipolar interactions in liquid nuclear magnetic resonance. J Chem Phys 1996;105:874-900.

[9] Vathyam S, Lee S, Warren WS. Homogeneous NMR spectra in inhomogeneous field. Science 1996;272:92-6.

[10] Warren WS, Ahn S. The boundary between liquidlike and solidlike behavior in magnetic resonance. J Chem Phys 1998;108:1313-25.

[11] Enss T, Ahn S, Warren WS. Visualization the dipolar field in solution NMR and MRI imaging: three-dimensional structure simulations. Chem Phys Lett 1999;305:101-8.

[12] Richter W, Lee S, Warren WS, He QH. Imaging with intermolecular multiple-quantum coherences in solution nuclear magnetic resonance. Science 1995;267:654-7.

[13] Warren WS, Ahn SD, Mescher M, et al. MR imaging contrast enhancement based on intermolecular zero-quantum coherences. Science 1998;281:247-51.

[14] Tsoref L, Shinar H, Seo Y, Eliav U, Navon G. Proton doublequantum filtered MRI-a new method for imaging ordered tissues. Magn Reson Med 1998;40:720-6.

[15] Mori S, Hurd RE, van Zijl PCM. Imaging of shifted simulated echoes and multiple spin echoes. Magn Reson Med 1997;37:336-40.

[16] Bifone A, Payne GS, Leach MO. In vivo multiple spin echoes. J Magn Reson 1998;135:33-6.

[17] Zhong J, Chen Z, Kwok E. In vivo intermolecular double-quantum imaging on a clinical 1.5 T MR scanner. Magn Reson Med 2000;43: 335-41.

[18] Zhong J, Kwok E, Chen Z. fMRI of auditory stimulation with intermolecular double-quantum coherences (iDQCs) at $1.5 \mathrm{~T}$. Magn Reson Med 2001;45:356-64.

[19] Chen Z, Lin GX, Zhong J. Diffusion of intermolecular zero- and double-quantum coherences in two-component spin systems. Chem Phys Lett 2001;333:96-102.
[20] Chen Z, Zhong J. Unconventional diffusion behaviors of intermolecular multiple-quantum coherences in nuclear magnetic resonance. J Chem Phys 2001;114:5642-53.

[21] Chen Z, Chen ZW, Zhong J. Quantitative study of longitudinal relaxation related to intermolecular dipolar interactions in solution NMR. Chem Phys Lett 2001;333:126-32.

[22] Zhong J, Chen Z, Zheng SK, Kennedy SD. Theoretical and experimental characterization of NMR transverse relaxation process related to intermolecular dipolar interactions. Chem Phys Lett 2001;350: $260-8$.

[23] Zhong J, Chen Z, Kwok E, Kennedy S. Enhanced sensitivity to molecular diffusion with intermolecular double-quantum coherences: implications and potential applications. Magn Reson Imaging 2001; 19:33-9.

[24] Zhong J, Chen Z, Kwok E. New image contrast mechanisms in intermolecular double-quantum coherence human MR imaging. J Magn Reson Imag 2000;12:311-20.

[25] Bowtell R, Gutteridge S, Ramanathan C. Imaging the long-range dipolar field in structured liquid state samples. J Magn Reson 2001; 150:147-55.

[26] Ramanathan C, Bowtell R. NMR imaging and structure measurements using the long-range dipolar field in liquids. Phys Rev E 2002;66:041201.

[27] Bouchard LS, Rizi R, Warren WS. Magnetization structure contrast based on imtermolecular multiple-quantum coherences. Magn Reson Med 2002;48:973-9.

[28] Capuani S, Branca RT, Alesiani M, Maraviglia B. Intermolecular double-quantum NMR techniques to probe microstructures on porous media. Magn Reson Imag 2003;21:413-4.

[29] Capuani S, Curzi F, Alessandri FM, Maraviglia B, Bifone A. Characterization of travecular bone by dipolar demagnetizing field MRI. Magn Reson Med 2001;46:683-9.

[30] Capuani S, Hagberg G, Fasano F, Indovina I, Castriota-Scanderbeg A, Maraviglia B. In vivo multiple spin echoes imaging of trabecular bone on a clinical 1.5 T MR scanner. Magn Reson Imag 2002;20: 623-9.

[31] Bowtell RJ, Bowley RM, Glover P. Multiple spin echoes in liquids in a high magnetic field. J Magn Reson 1990;88:643-51.

[32] Minot ED, Callaghan PT, Kaplan N. Multiple echoes, multiple quantum coherence, and the dipolar field: demonstrating the significance of higher order terms in the equilibrium density matrix. J Magn Reson 1999;140:200-5.

[33] Charles-Edwards GD, Payne GS, Leach MO, Bifone A. Effects of residual SQCs on signal contrast in iMQC studies. Proc Intl Soc Magn Reson Med 2003;11:1109. 